\title{
Modelling of complex fenestration systems - application of different toolchain approaches on real case scenarios
}

\author{
Martin Hauer ${ }^{1,3}$, Giuseppe De Michele ${ }^{2}$, Francesco Babich², Daniel Plörer ${ }^{3}$ Stefano Avesani ${ }^{2}$ \\ ${ }^{1}$ Bartenbach GmbH, Aldrans, Austria \\ ${ }^{2}$ EURAC Research, Bolzano, Italy \\ ${ }^{3}$ University of Innsbruck, Unit for Energy Efficient Buildings, Innsbruck, Austria
}

\begin{abstract}
This paper presents a comparative study among wellestablished simulation tools showing their capabilities evaluating complex fenestration systems in terms of thermal and daylight performance. Two powerful and flexible toolchain approaches based on EnergyPlus and TRNSYS including third-party software are analysed in detail and their application is shown on exemplarily test cases. By investing into comparative simulations, capabilities of both toolchains are highlighted, and pros and cons are discussed within this paper. Upon comparison with measured data, the ability of both toolchains to predict real case scenarios is shown. Satisfying alignment has been achieved in energy performance prediction (cooling loads, temperatures) as well as daylighting prediction (illuminance levels). Remaining uncertainties reflects the sensibility in used model definitions, used data as well as tool handling.
\end{abstract}

\section{Introduction}

\section{The role of CFS modelling}

Trends in implementing complex fenestration systems (CFS) in the modern architecture of non-residential buildings are driving the need for improved methods and validated tools for planning and engineering purposes. Especially for highly glazed building facades, the detailed modelling of CFS plays a major role in enabling reliable simulations for thermal and daylighting performance predictions as well as for comfort evaluation.

Models development to evaluate CFS within building energy simulation tools has increased significantly in recent years (Kirimtat et al. 2016). Although the number of tools is increasing, workflows including important aspects like high modelling flexibility, usability and efficient runtime while preserving detailed results are still rarely available - particularly in the field of CFS modelling (Loonen et al. 2016).

Numerous case studies are published in literature, examining the potential of CFS under various climate conditions and room scenarios (Gong et al. 2016; Santos et al. 2018; Bustamante et al. 2015). However, clear and proved workflows in how to gain the required model information as well as how to setup a simulation model for a coupled thermal and daylighting analyse of complex facades are still rare.
At the same time the utilization of daylight in buildings has gained a significant relevance in reducing the electrical energy demand for artificial lighting as well as optimizing the overall energy demand for heating and cooling (Pyonchan et al. 2009), besides the fundamental role in internationally renown certification protocols such as LEED, BREEAM, and WELL. Evaluating building façade systems in the early stage of design is crucial in order to meet low energy requirements and highest comfort levels in the operation.

\section{Overview on simulation tools for CFS evaluation}

The challenge tackled in this paper is to define a reliable workflow to evaluate the performance of CFS. Nowadays, several simulation tools exist to perform dynamic energy and daylighting simulations for CFS. These tools rely on complex models, which require deep knowledge and simulation skills by the user and often do not offer a cosimulation environment. Moreover, either for the part of daylight simulation as well as the part of thermal simulation of CFS, simplifications are made in the modelling to enable a numerical representation with a reasonable effort in providing input data, model setup and simulation runtime.

For this reason, the use of a trustworthy procedure to simulate CFS and the awareness of the likely error that could occur by using such detailed models are essential for a conscious design of energy efficient buildings. In (Loonen et al. 2016) the current status, requirements and opportunities on evaluating adaptive facades with the most powerful and validated simulation tools (EnergyPlus, ESP-r, IDA-ICE, IES VE and TRNSYS) are shown. Based on this work, two of the mentioned software packages have been analyzed checking their capabilities from the point of view of a coupled energy and daylight evaluation of CFS.

In the last years, several widely used building simulation tools have extended their ability to perform coupled daylight and thermal evaluations for CFS based on Radiance Three-Phase Method (3PM) (Saxena et al. 2010) and the ISO15099 standard (EN ISO 15099). Even though most of the methods claim to enable an integrated approach to increase the overall efficiency concerning daylight and energery-related aspects, just few of them allow timestep-based feedback loops between the thermal and daylight simulation routine. However, this is a crucial aspect to design comfortable and efficient buildings, e.g. 
for developing improved control strategies and optimizing thermal and visual aspects of a complex façade systems in detail.

For the EnergyPlus simulation kernel, serveral graphical user interfaces (GUIs) exist, most of them open source and non-commercial. The probably most widely used is OpenStudio (v.2.7), which enables an energy-, daylightand glare evaluation of CFS using the 3PM (Guglielmetti 2015). Nevertheless, the functionality for CFS is limited because it does not allow to insert user-defined bidirectional scattering distribution functions (BSDFs) for thermal and daylight calculations as well as no adaptive shading states according to daylight and thermal conditions. Instead of a timestep-based coupling, OpenStudio precalculates annual illuminance schedules for each window group (windows, which are equally controlled) as well as for the control of a simplified shading (Fc-factor). In this sense, a powerful and flexible approach is connecting EnergyPlus via using the Grashopper plugins Ladybug and Honeybee with Rhinoceros. Beside the Radiance Five-Phase Method (Lee et al. 2018) it also enables userdefined BSDFs for the thermal and daylight calculation. However, a compiled energy and daylight optimization on timestep basis is still not implemented.

IDA ICE (version 4.7 and higher) allows the simplified calculation of daylight factors and illuminance values for several thermal zones, userdefined sensor grids as well as different sky models. By (Karlsen et al. 2015) a method is presented to couple IDA ICE with Radiance using the 3PM. Nevertheless, a timestep-based coupling between thermal and daylight evaluation is also still not possible.

IES VE and ESP-r enable simplfied daylight calculations based on the radiosity method as well as rayracing technique by using Radiance. Also there, the integrated daylight module is rather for calculating the daylightresponding artifical light demand instead of detailed coupled simulations. Algorithms for detailed CFS analysis are not included.

TRNSYS involves since version 18 additional daylighting functionalities within Type56 using Daysim. Additionally it enables a detailed thermal modelling of the CFS using the latest model implementations based on a combination of bidirectional scattering distribution functions (BSDFs) and the ISO15099 standard (Hiller and Schöttl 2014). The Grasshopper-based platform TRNLizard enables parametric modelling with a focus on coupled thermal and daylight analysis (Frenzel and Hiller 2014).

Nevertheless, also in Type56 daylight calculations are primarily used for calculating the daylight-responding artificial light demand as well as enabling dynamic shading strategies based on a simplified Fc-factor.

To perform a compiled thermal and daylight analysis of CFS, the Artlight routine was developed and implemented as a new TRNSYS-Type (Hauer and Geisler-Moroder 2017). Artlight showed first and with success the capability of a time-step based coupling of thermal and daylight simulations. In combination with the new Type56-BSDF model, TRNSYS with Artlight enables an accurate evaluation of shading elements and daylight redirecting components in terms of visual and thermal performance, paired with the development of enhanced control strategies.

A major reason that most tools come up with a scheduled model input instead of instantaneous timestep coupling is due to time and data intensive simulation effort, as the 3PM workflow has to be executed at least once within each simulation timestep. The loss in flexibility by precalculated illuminance values and shading states has to be accepted thereby.

\section{Toolchain approach for coupled thermal and daylight evaluation of CFS}

Among all tools which underwent screening, EnergyPlus and TRNSYS are the tools, which are most widely used and provide the necessary functionalities to perform a coupled thermal and daylight evaluation. Based on these tools, two toolchain workflows have been defined starting from the shared geometry platform Rhinoceros. The free available Grasshopper plugins Ladybug and Honeybee connect EnergyPlus and Radiance, while TRNLizard in combination with Artlight connects TRNSYS with Radiance. The geometrical modeling is done in Grashoopper, the model set up as well as the transition into the simulation input files to perform the simulations in EnergyPlus (*.idf) and TRNSYS (*.d18, *.b18) has been implemented via Grashopper.

\section{Shared geometry platform}

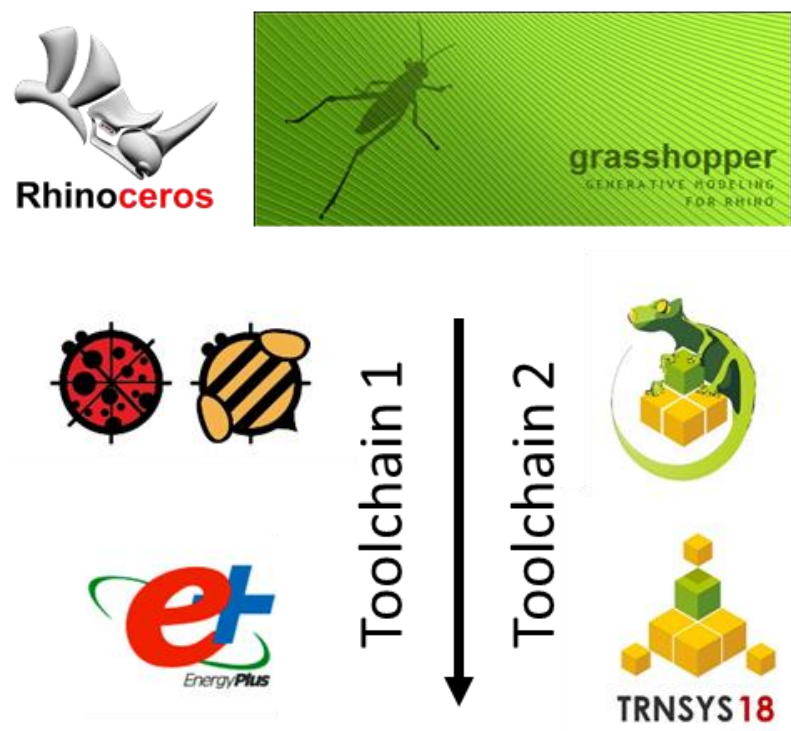

Figure 1: Toolchain workflow for evaluating CFS

\section{Simulation workflows}

In the following subsections, the modelling capabilities of the tools involved in both toolchains are explained in detail and compared. Moreover, the thermal-daylighting interaction capabilities are discussed. 


\section{Toolchain 1: Rhinoceros - TRNLizard/Artlight - TRNSYS}

Since TRNSYS18, a free plug-in for Grasshopper named TRNLizard is released, which allows to perform parametric thermal and daylight simulations based on a 3D-geometry in Rhinoceros. It combines the advantages of the parametric architecture from Grasshopper tools with the powerful solving engine of TRNSYS kernel. The modular structure of TRNLizard provides a greater flexibility compared to the classical Simulation Studio interface. Starting from a pre-defined template for singleor multizone simulation, it also allows to include userdefined components (Types). Within this tool comparison, Rhinoceros is used as geometrical platform to setup the geometrical model and the TRNLizard plugin is used to setup the energy model for the thermal simulation. For this study, Artlight was implemented as user-defined component in TRNLizard.

With Artlight, a coupling of the thermal modelling in TRNSYS with the daylight modelling in Radiance is realised. The daylight simulation routine is based on the Radiance 3PM, while the detailed thermal modelling of the CFS is done using the latest model implementations in Type56 within TRNSYS 18.

Artlight is linked with the radiation processor (Type15) for the environmental input data as well as with the multizone building model (Type56) for the coupled daylight and thermal modelling. The structure of the Artlight-routine is shown in Figure 2.

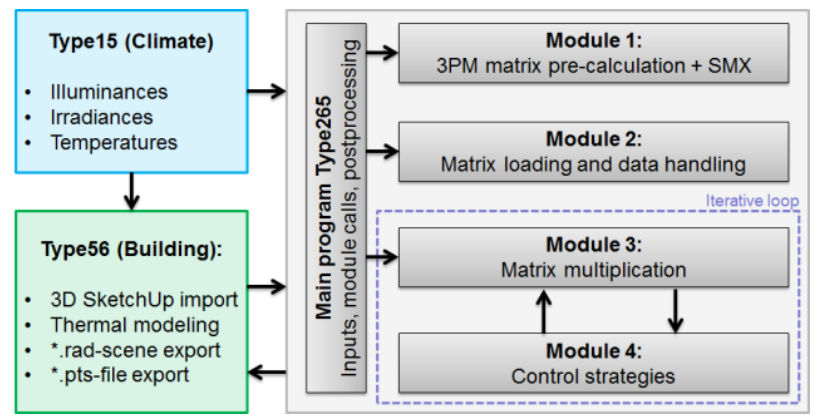

Figure 2: Structure and input-output of Arlight

Module 1 can either use the exported files from Type56 or a manually created Radiance scenes to generate the view matrices and the daylight matrices. As a default, the daylight matrix is calculated using the Reinhart MF:4 subdivision of the Tregenza sky (2306 patches).

The view matrix has to be calculated separately for each window part and for each orientation. Using Radiance tools, a user-provided *.epw-file can be converted via the *.wea-file format into a sky matrix (*.smx). The BSDF data for the transmission matrix has to be provided separately for each window subdivision and sets of blind positions by the user. They can be generated with external tools (WINDOW7, genBSDF).
To enable a fast calculation, all necessary matrix data to perform the daylight simulation has to be loaded into the RAM before the first simulation timestep. The data loading, caching and flexible storage (re-)allocation depending on the actual simulation setup is done in Module 2.

$Z=\left[\begin{array}{c}1 \\ . . \\ 2306\end{array}\right]^{T} *\left[\begin{array}{cc}1 & 145 \\ . . & . . \\ 2306 & . .\end{array}\right] *\left[\begin{array}{cc}1 & 145 \\ . & . . \\ 145 & . .\end{array}\right] *\left[\begin{array}{cc}1 & s \\ . & . . \\ 145 & . .\end{array}\right]$

In Module 3, the whole 3PM matrix multiplication given in eq. (1) is processed in a repeating loop depending on the number of window (subdivisions) and façade orientations. As a result, the timestep-based illuminance and luminance values are analysed, if they achieve the given threshold criteria shown in eq. (2) and eq. (3). Index number "s" represents the number of sensor points.

$$
\begin{aligned}
& \text { Illuminance: } \left.\left[\frac{\sum_{i=1}^{S} E_{i}}{s} \geq 500 l x\right)\right] \stackrel{\text { then }}{\longrightarrow} \text { true } \\
& \text { Luminance: }\left[\sum_{i=1}^{s}\left(L_{s, o} \leq 5000 \frac{\mathrm{cd}}{\mathrm{m}^{2}}\right)\right] \stackrel{\text { then }}{\longrightarrow} \text { true }
\end{aligned}
$$

Different implemented control strategies further allow running the coupled simulation either focussing on thermal optimization or daylight optimization of the situation in the room.

\section{Toolchain 2: Rhinoceros -Honeybee - Energy Plus}

Honeybee [+] is an improved version of Honeybee legacy, which allows an extensive analysis of daylighting performances of CFS. In fact, the tool includes several matrix methods of Radiance (Subramaniam, 2017), and allows to employ BSDF with different resolutions (i.e. Klems and Tensor-tree) as well as a 3D geometry of the shading device. Honeybee[+] offers a default control strategy based on interior illuminance level, either global or direct. Other controls can be defined by modifying the python code of the dynBlindSchd component, but this requires a coding capability by the user. Alternatively, a csv file with the shading configuration can be addressed. Concerning the thermal modelling, even if EnergyPlus allows for analysis of CFS by means of ISO15099 coupled with BSDFs for the optical part, it is not possible to directly use this module in the Honeybee components.

A customization of the idf file is required to import the BSDF into Honybee and activate the Complex Fenestration State object. This procedure includes two steps:

1. From the idf file containing the BSDFs and the other fenestration system information, the section Construction:ComplexFenestrationState has to be extracted, and connected as EPconstruction to the window component. 
2. The remaining information in the idf file has to be set as additionalString input to the runEnergySimulation component.

The control of the "Complex Fenestration State" can be done through the EMS (Energy Management System) object within EnergyPlus. Nevertheless, the EMS has not been yet implemented in Honeybee, this lack limits the applicability of the tool for dynamic CFS that require BSDF data to be characterized. If standard shading systems are employed (i.e. venetian blind, roller shade, performed mesh and switchable glazing), the several shading control types of EnergyPlus can be set in the EPWindowShades component.

An interaction between thermal and daylighting physics is not possible during simulation run-time. Information is shared as schedule after one of the simulations has completed. In particular, energy and daylighting simulations can share schedules for the shading activation and daylight-responding artificial light demand. In this sense, Honeybee is user-friendly since the user is not required to manipulate or post-process the data from one simulation to the other.

Nevertheless, this functionality prevents the possibility to define multi-objective control strategies that involve simultaneously thermal and daylighting parameters, such as interior air temperature and illuminance values.

\section{Simulation test cases}

In this section, the simulation test cases are described. To check both toolchain approaches against each other, two major comparative settings have been defined: (1) on a theoretical basis with a shared geometry model and (2) an individual comparison of both toolchain workflows against measured data.

\section{Geometry model: PASSYS cell}

For the theoretical model comparison, a geometrical box model representing the PASSYS outdoor test stand at University of Innsbruck (ref. Figure 3) has been implemented in the toolchains and used for the comparison against real-case measurements. The test façade is characterised by a daylight redirecting system.

The geometry represents an office box with the dimensions of $4.98 \mathrm{~m} \times 2.77 \mathrm{~m} \times 2.73 \mathrm{~m}$. The southoriented façade involves three façade areas (FA) including an opaque part (FA1) and two transparent façade parts in the middle (FA2) and the top (FA3) of the facade. Both parts can be equipped with different shading systems and can be also controlled independently.

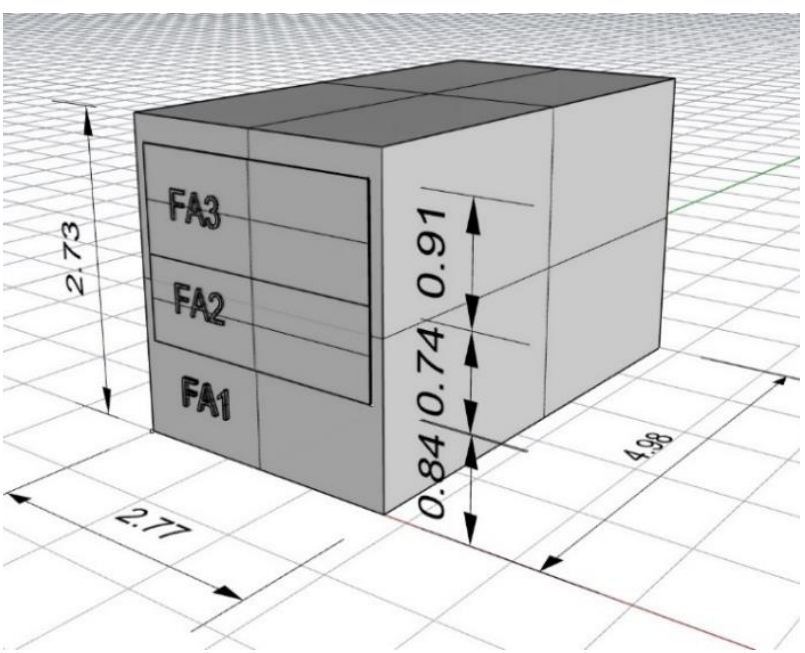

Figure 3: Sketch of the PASSYS test cell model in Rhinoceros

\section{Theoretical comparison}

For the theoretical comparison of both proposed simulation toolchains, the PASSYS box was modelled with constructions for walls, roof and ceiling based on theoretical values from literature. All surfaces are modelled with external boundary conditions (wind and sun exposed) except the backside wall, which is modelled adiabatic towards the adjacent service room. Other settings like ventilation, infiltration and internal gains have been not modelled in order to reduce modelling influences. Heating and cooling set points are set constantly to $20^{\circ} \mathrm{C}$ respectively $26^{\circ} \mathrm{C}$. For the simulated location of Innsbruck, the EnergyPlus weather file (*.epw) from EnergyPlus website is used.

\section{Measurement comparison}

For the real case comparison, monitoring data from the PASSYS outdoor test stand from two separated test phases, including a shaded and non-shaded façade setting, are used. Climatic data is available in a 3-minute interval from the weather station on site including ambient dry bulb temperature and incident radiation in horizontal and vertical (south) orientation - separated into a direct and diffuse component.

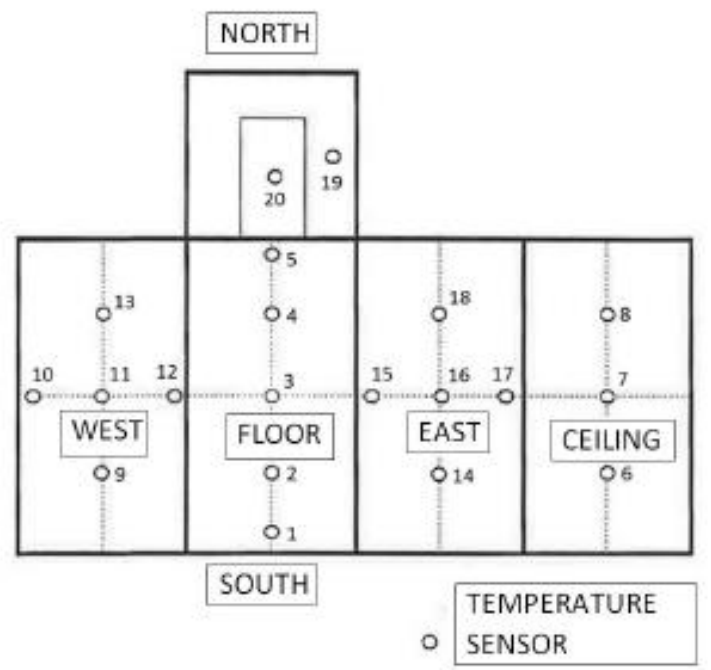

Figure 4: Sensors installed in the PASSYS cell envelope 
For the thermal model comparison monitoring data is evaluated for inside and outside surface temperatures of walls, floor and roof, room (ref. Figure 4), air temperatures in 3 different height levels as well as power loads for heating, cooling and internal gains.

For the daylighting comparison, two horizontal illuminance sensors positioned along the centreline of the room towards the room depth with $1.5 \mathrm{~m}$ (near) and $4 \mathrm{~m}$ (far) distance from the façade installed.

\section{Installed test façade}

During the monitoring phase, a closed cavity façade with two separate venetian blind systems (ref. Figure 5) was installed in between of an impingement glazing and a 3-pane insulation glazing unit towards inside.

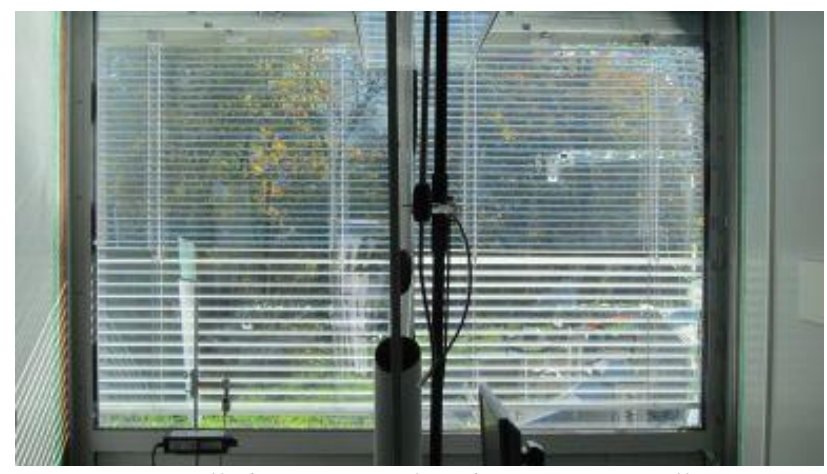

Figure 5: Installed monitoring facade at PASSYS-cell

The measurements dataset used for the comparison refers to two façade configurations. In the configuration $\mathrm{nr}$. 1, the facade is not shaded (retracted blinds in FA2 and FA3). For configuration nr. 2 the façade shading system is deployed: FA2 is equipped with a downwards curved, diffuse shading blind, FA3 is equipped with an upwards curved, highly-reflective blind forcing specular daylight reflection towards the depth of the room.

Table 1: Monitoring facade setup (WINDOW7)

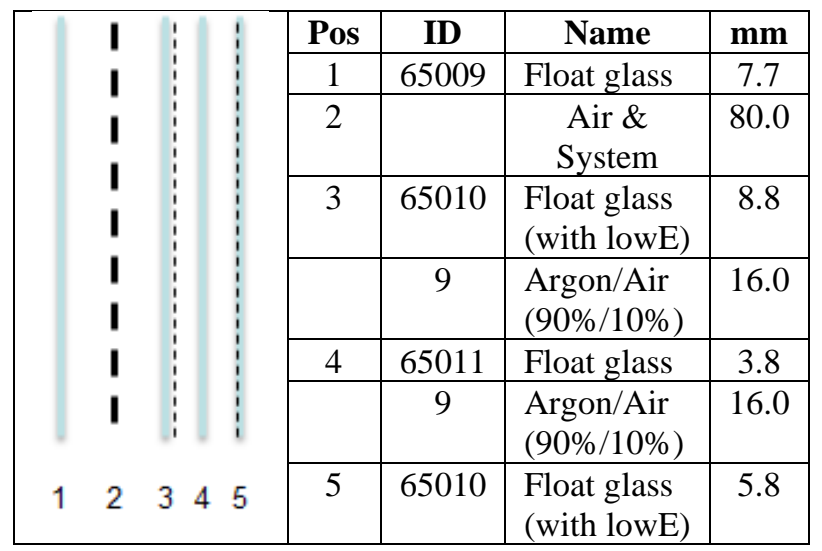

\section{Simulation model setup}

In this section, the detailed setup of the façade model within both simulation toolchains is described as well as the fit of the thermal cell model to match the real-case situation of the PASSYS cell.

\section{Facade modelling}

For the detailed thermal modelling of CFS within the multizone building model, both investigated toolchain workflows use similar procedures. The standard window model in TRNSYS bases on a one-dimensional angular dependent value for transmission, reflection and absorption. TRNSYS 18 comes up with a new CFS model based on BSDF and ISO15099 (Hiller und Schöttl 2014), which enables a detailed modelling of the multiple scattered reflection and transmission especially in case of specular daylighting systems.

In both TRNSYS and EnergyPlus, the modelling is separated into shortwave radiation modelling by the pre-calculated BSDF data and the interrelated longwave radiation modelling according to algorithms defined in the ISO15099. This standard is currently the most comprehensive and widely-used modelling standard for complex glazing systems incorporating blinds.

To gather the system data for the model definition in TRNSYS and EnergyPlus, WINDOW7 was used as thirdparty and free-to-use tool from LBNL as a shared data base to describe the optical glazing properties, using entries from the IGDB (ref. Table 1). For modelling the shading blinds in detail, the genBSDF tool within Radiance was used to simulate a Klems BSDF based on Geometry and Material description of the blind (ref. Table 2).

By importing the created BSDF into WINDOW7 as user defined shading layer, an overall system BSDF was created by combining it with the glazing layers.

Table 2: Specification of used blind systems

\begin{tabular}{|l|l|l|}
\hline Parameter & \multicolumn{1}{|c|}{ System - FA2 } & System - FA3 \\
\hline width & $60 \mathrm{~mm}$ & $60 \mathrm{~mm}$ \\
\hline spacing & $52 \mathrm{~mm}$ & $34 \mathrm{~mm}$ \\
\hline rise & $4.6 \mathrm{~mm}$ & $6 \mathrm{~mm}$ \\
\hline$\varepsilon_{\text {front }}$ & 0.88 & 0.04 \\
\hline$\varepsilon_{\text {back }}$ & 0.88 & 0.88 \\
\hline material $_{\text {front }}$ & RAL9016 & Miro3 \\
\hline material $_{\text {back }}$ & RAL9016 & RAL9016 \\
\hline
\end{tabular}

\section{PASSYS cell model fit to measured data}

In order to calibrate the theoretical model with the real PASSYS cell, a measurement period of two weeks (calibration phase) was used to match the construction setup with the real conditions. By using GenOpt, the thicknesses of the different construction layers were varied by using the measured room temperature as target function for the optimization process. By this, the overall 
U-value of the envelope as well as their thermal capacitance were adjusted to minimize the difference.

The boundary conditions of the PASSYS cell were measured over a period of two weeks ( $4^{\text {th }}$ of January 2016 to $18^{\text {th }}$ January 2016). The parameter identification problem optimizes the root mean squared value of the overall difference between measured data (ref. Figure 4 with inside surface temperature of 5 envelope surfaces and air temperature) and simulated data.

For building performance, the analysis error can be defined as shown in equation (4) by the difference between a predicted value and a measured value. In the present case, the error is calculated for summation of the indoor air temperature and inside surfaces temperature averaged in the test room.

$R M S D=\sqrt{\frac{\sum_{i=1}^{n}\left(m a_{i}-s a_{i}\right)^{2}+\sum_{i=1}^{n} \sum_{j=1}^{5}\left(m s_{j i}-s s_{j i}\right)^{2}}{n}}$

$m a_{i}=$ measured air temperature

$s a_{i}=$ simulated air temperature value

$m s_{j i}=$ measured inside surface temperature for different wall orientations

$S S_{j i}=$ simulated inside surface temperature for different wall orientations

To solve the parameter identification, hybrid generalized pattern search algorithm with particle swarm optimization algorithm was used. Using this algorithm, the cost function mentioned in the previous section was parametrized.

The parametric identification of this model reduces the thermal capacitance of air by $20 \%$. On the other hand, insulation thickness of the insulation material was decreased on most of the wall except the ceiling. The upper bound of the ceiling thickness is $0.4 \mathrm{~m}$ in the literature and reached the peak at the end of the optimization process. This is because the ceiling was entirely exposed to solar radiation of the PASSYS-cell assuming that heat gain through ceiling was greater compared to the other wall constructions. Wall to service and floor experiences the maximum decrement in terms of thickness of the insulation layer, almost $50 \%$ in both cases. As the PASSYS cell is lifted above from the ground by pillows, the outside surface of the floor is not in contact with the ground. Besides, thickness of concrete layers with high thermal mass have been also decreased to reduce the overall thermal capacitance of the model.

After the optimization process a cross-check period was used to validate the optimized model. The correspondence between measured and simulated room air temperature for the cross-check period reached a remaining root mean square deviation of $0.98 \mathrm{~K}$.

\section{Simulation results and analysis}

In the following subsection, the comparison between the theoretical model of both toolchain approaches and the comparison of the simulation results with measured data are reported.

\section{Theoretical model results}

In a first step, both toolchain approaches are tested based on the theoretical PASSYS settings, using the literature values for the construction layers. Also, for the façade model setup, the same set of data was used according to the description in the previous chapter.

Two cases have been evaluated: for case 1, the theoretical PASSYS model was modelled using external boundary conditions for all walls (except the northern wall, which is adjacent to a service room). Beside this, the convective heat transfer coefficients for all surfaces (inside and outside) are calculated in EnergyPlus and used as input in TRNSYS. Physical parameters like solar absorption and longwave emissivity are set equally.

Table 3. Case 1 - Ideal heating and cooling loads

\begin{tabular}{|c|c|c|c|c|}
\hline Case 1 & \multicolumn{2}{|c|}{ EnergyPlus } & \multicolumn{2}{c|}{ TRNSYS } \\
\hline & heating & cooling & heating & cooling \\
\hline $\mathrm{Wh} / \mathrm{m} 2$ & 10340.3 & 8646.59 & 12725.56 & 10461.29 \\
\hline rel. dev. & $20.68 \%$ & $18.99 \%$ & & \\
\hline
\end{tabular}

For case 1, the results show significant differences of $20.68 \%$ for heating and $18.99 \%$ for the cooling energy demand between both toolchains. TRNSYS estimates clearly higher demand compared to EnergyPlus.

To improve this relevant deviation, in case 2 the surface outside temperatures of all outdoor walls (except the south façade) - calculated by EnergyPlus - have been used as temperature boundary condition input for the external walls in TRNSYS. By this approach, the differences in the results between both tools have been reduced significantly for the heating demand to $5.29 \%$ and for the cooling demand to $0.54 \%$. Therefore, the model setting of case 2 is used for further theoretical analysis.

Table 4. Case 2 - Ideal heating and cooling loads

\begin{tabular}{|c|c|c|c|c|}
\hline Case 2 & \multicolumn{2}{|c|}{ EnergyPlus } & \multicolumn{2}{c|}{ TRNSYS } \\
\hline & heating & cooling & heating & cooling \\
\hline $\mathrm{Wh} / \mathrm{m} 2$ & 10272.06 & 8646.60 & 10833.02 & 8601.29 \\
\hline rel. dev. & $5.29 \%$ & $0.54 \%$ & & \\
\hline
\end{tabular}

For case 2, Figure 6 shows the match for the free-floating room temperature between both toolchain approaches. In a yearly trend, slightly higher deviations have been shown in winter months (October - March). In general, the value for the rRMSE based on a yearly simulation is $4.72 \%$. Beside the room air temperature, also the operative room air temperature corresponds well between both tools. 


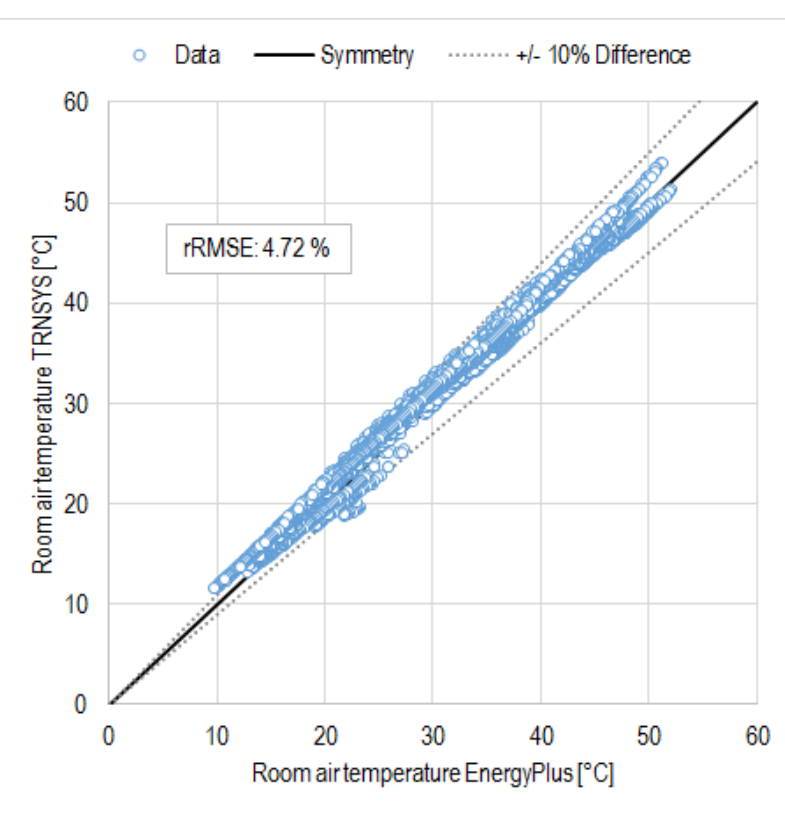

Figure 6: Comparison of sensitive room air temperature

A similar comparison between both toolchain approaches is shown in Figure 7 for the resulting inside surface temperature of the glazed façade. Except of some particular higher deviations, the rRMSE based on the yearly simulation result is again low with $4.78 \%$.

For this comparison, in EnergyPlus the spectral window model is used, while in TRNSYS the new Type56 BSDF model is used. Switching in TRNSYS to the DOE-model caused higher deviations in the results.

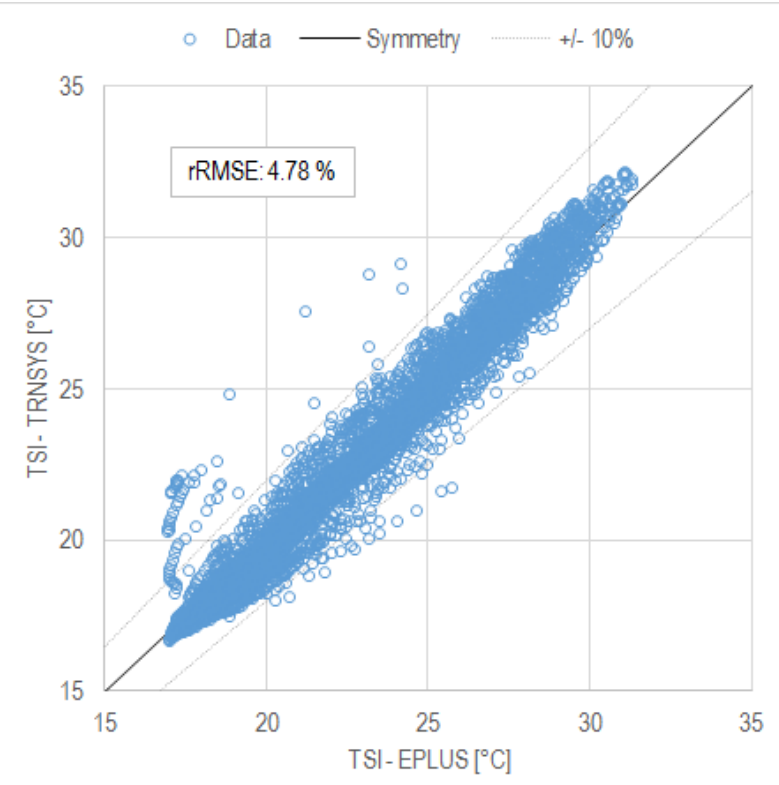

Figure 7: Comparison of inside glazing surface temperatures for the glazed situation

In Table 5 minimum, maximum and mean values are shown for the inside glazing surface temperature. For the case without blinds, FA2 and FA3 shows identical results through the same setting. The mean values correspond well between both toolchain approaches with $0.1^{\circ} \mathrm{C}$.
Also, the differences for the maximum and minimum value lower than $1^{\circ} \mathrm{C}$.

Table 5: Statistical values for the glazed situation of the theoretical model comparison

\begin{tabular}{|l|c|c|c|c|}
\hline & \multicolumn{2}{|c|}{ EnergyPlus } & \multicolumn{2}{c|}{ TRNSYS } \\
\hline & FA2 & FA3 & FA2 & FA3 \\
\hline $\operatorname{Max}\left[{ }^{\circ} \mathrm{C}\right]$ & 31.31 & 31.31 & 32.15 & 32.15 \\
\hline Min $\left[{ }^{\circ} \mathrm{C}\right]$ & 16.93 & 16.93 & 16.69 & 16.69 \\
\hline Mean $\left[{ }^{\circ} \mathrm{C}\right]$ & 22.75 & 22.75 & 22.65 & 22.65 \\
\hline
\end{tabular}

For the comparative case with blinds in both façade areas, the correspondence of the inside glazing surface temperature between both toolchain approaches is shown in Table 5 and Figure 8. Both tools are using the integrated BSDF model including the ISO15099 standard for the complex glazing modelling. EnergyPlus shows slightly higher temperatures in this case then TRNSYS.

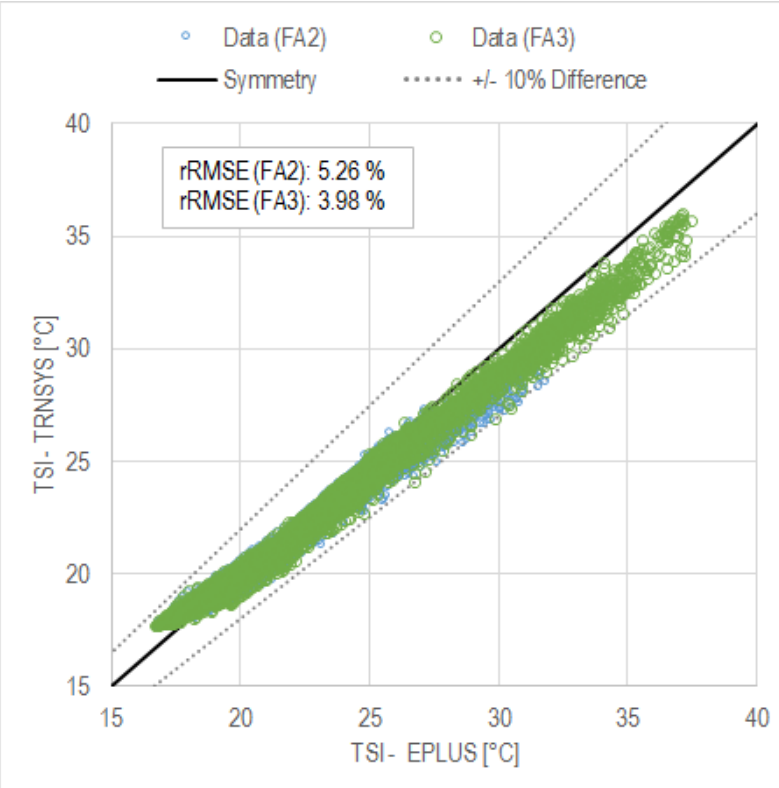

Figure 8: Comparison of inside glazing surface temperatures for the shaded situation

The maximal and minimal temperature between both toolchain approaches differs now up to $2^{\circ} \mathrm{C}$ for both façade parts. Nevertheless, the mean temperature value is within $0.5^{\circ} \mathrm{C}$ difference. Also the rRMSE for both blind parts are low with $5.26 \%$ respectively $3.98 \%$.

Table 6: Statistical values for the shaded situation of the theoretical model comparison

\begin{tabular}{|l|c|c|c|c|}
\hline & \multicolumn{2}{|c|}{ EnergyPlus } & \multicolumn{2}{c|}{ TRNSYS } \\
\hline & FA2 & FA3 & FA2 & FA3 \\
\hline Max $\left[{ }^{\circ} \mathrm{C}\right]$ & 32.80 & 37.49 & 30.88 & 35.96 \\
\hline Min $\left[{ }^{\circ} \mathrm{C}\right]$ & 16.76 & 16.74 & 17.61 & 17.64 \\
\hline Mean $\left[{ }^{\circ} \mathrm{C}\right]$ & 23.18 & 23.80 & 22.81 & 23.45 \\
\hline
\end{tabular}


For the theoretical model comparison, hourly illuminance values calculated by Artlight (TRNSYS) and Honeybee (EnergyPlus) are compared. As both algorithms base on the Radiance 3PM and use the same input matrices (SMX, DMX, BSDF, VMX), similar results have to be expected. As shown in Figure 9, the daily profile for the $1^{\text {st }}$ of January and for the shaded situation shows a perfect correspondence of the results.

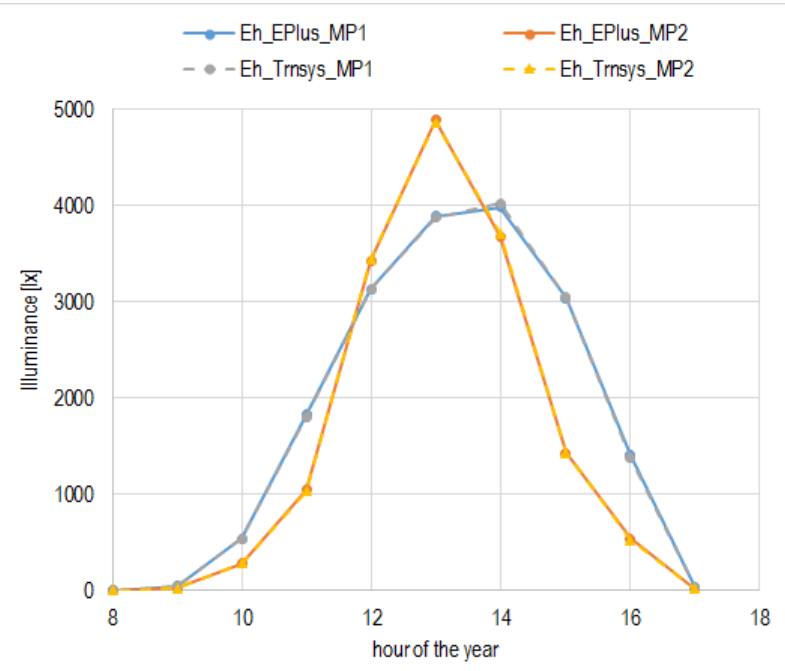

Figure 9: Comparison of illuminance results for the shaded situation

\section{Comparison against measured data}

For the comparison against measurement, the box model of the PASSYS cell from the previous theoretical comparison was overtaken. The box was again modelled with outside surface temperature as boundary condition. In particular, the measured outside surface wall temperatures from the monitoring phase were used, which also corresponds to the model setup during the optimisation process. The south wall including the testing façade had to be modelled as external wall including solar radiation and temperatures.

The radiation measurements were available for the global and diffuse part on the horizontal (situated next to the PASSYS cell) as well as on the vertical (directly next to the measuring façade). EnergyPlus radiation input mode is restricted only to the horizontal one (direct-normal, diffuse), while TRNSYS allows to use vertical measured radiation data (global, diffuse) as input for Type56. EnergyPlus is using the Perez model to calculate the vertical radiation, while for TRNSYS directly the vertical measured radiation is used. In Figure 10, a comparison of the global radiation measured in the façade compared to the calculated one by EnergyPlus is reported. It shows a reasonable accordance for the comparative purpose with an rRMSE of $4.18 \%$ for the measurement period 1 (without blinds).

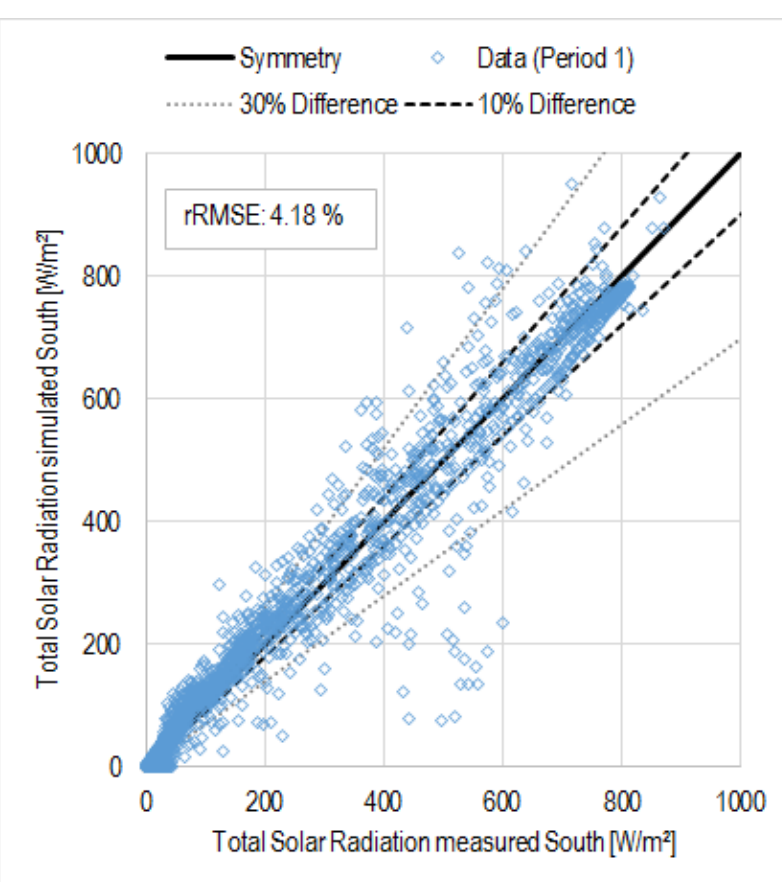

Figure 10: Comparison of the solar radiation input for the testfacade

Also for the comparison with the measurements, a case without shading (gathered blinds) and a case with active shading (e.g. FA2: shading blinds with $0^{\circ}$, FA3: redirecting blinds with $0^{\circ}$ ) has been analysed. In Figure 11 the total transmitted solar radiation values through both façade areas are compared. TRNSYS shows slightly higher transmittance values in the morning and evening hours, while the daily peaks correspond well.

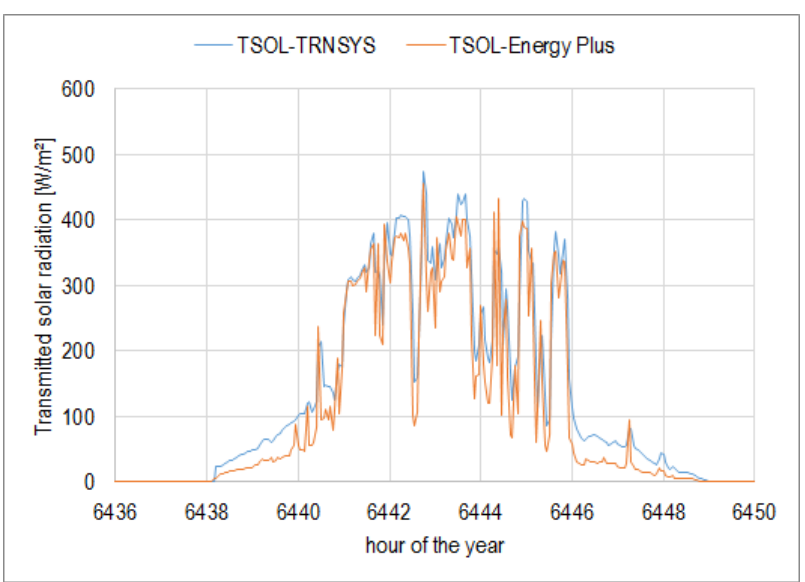

Figure 11: Transmitted solar radiation for the glazed situation

In Figure 12 the same comparison is shown for the total solar transmission in the case with blinds. Significant differences in the instantaneous values between EnergyPlus and TRNSYS occurs. This is because the thermal BSDSF model in TRNSYS interpolates between the neighbour Klems-patches, while EnergyPlus takes the actual value of the patch hit by the sun. Nevertheless, the integral values of the day are similar. 


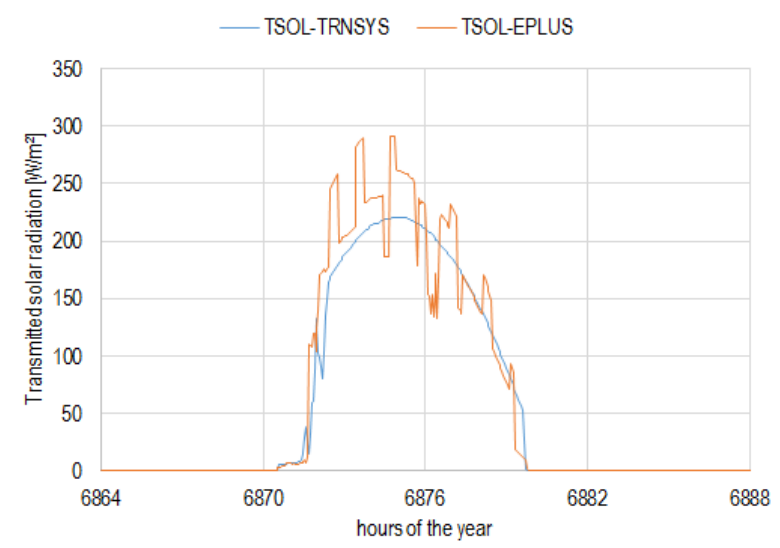

Figure 12: Transmitted solar radiation for the shaded situation

The comparison of measured and simulated illuminance values shows that a satisfying correspondence could be found for days with diffuse conditions. The daylight models used are set as the ones for the theoretical comparison. The check against measurements is done only considering TRNSYS and Artlight, because the daylight algorithms in both tools (TRNSYS and EnergyPlus) are identical. In Figure 13, the comparison between simulated and measured results (Case 1: only glazing) show a good agreement with an rRMSE of $10.77 \%$ for the façade near measurement point (MP1) and an rRMSE of $5.46 \%$ for the façade far measurement point (MP2). For MP1, the simulation is slightly overestimating the illuminance values compared to the measurement

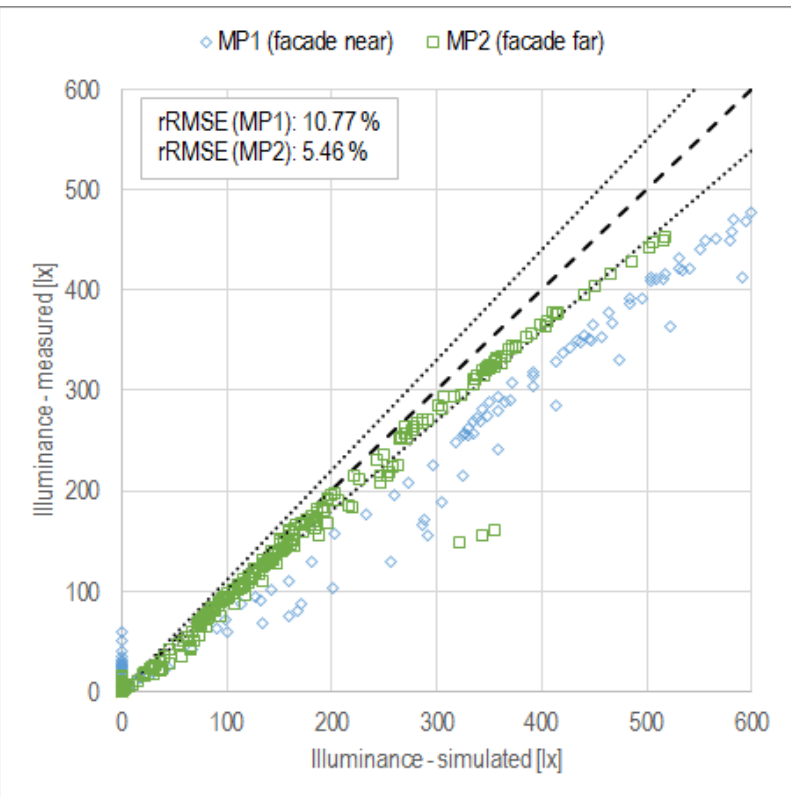

Figure 13: Comparative illuminance values - glazed situation

Also for case 2 (FA2: shading blind, FA3: redirecting blind) the results are satisfying for both measurement points (ref. Figure 14). For both cases, at days with high parts of direct solar radiation the simulation clearly overestimated the illuminance values compared to the measurement. This reason behind this is still under investigation, although it seems to be strongly connected to errors in the measurement of the direct and diffuse radiation components.

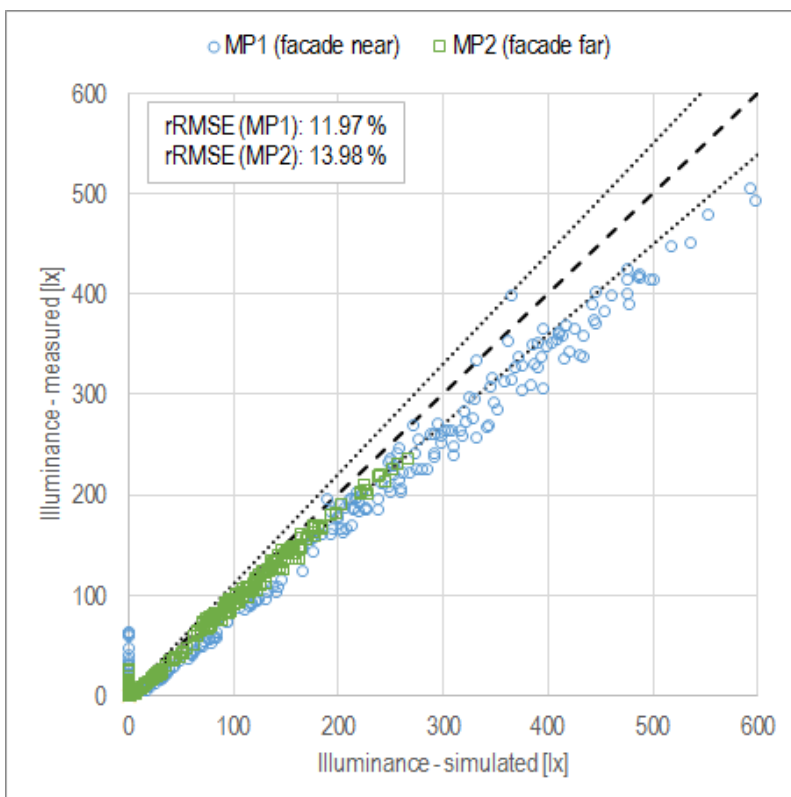

Figure 14: Comparative illuminance values - shaded situation

For comparing the simulated and measured cooling load during the operation of the PASSYS cell, the measured room air temperature was used as set point in the simulation, which was targeted within each time step by setting ideal heating and cooling in the simulations.

Figure 15 shows a 2-days trend of the instantaneous values (3min interval) as well as the averaging hourly trend of the cooling loads for the glazing situation. It includes the measured values during the PASSYS operation as well as the simulated cooling loads by TRNSYS and EnergyPlus.

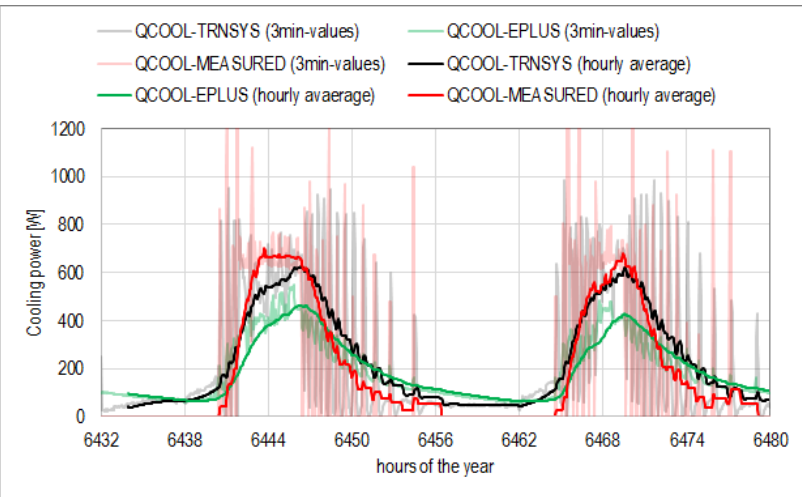

Figure 15: Instantaneous cooling load - glazed situation

The high peak values are resulting from continuous onoff operation mode of the cooling unit, caused by the oversizing of cooling system installed in the PASSYS cell. Similarly, TRNSYS reacts by assuming an ideal cooling system. A detailed comparison of the instantaneous cooling peaks shows a good agreement between simulated values in TRNSYS and measurement. 
On the other hand, EnergyPlus does some averaging of the output values, which reduces the peaks in the resulting cooling load.

In general, the simulated values in TRNSYS are closer to the measured results, while EnergyPlus underestimates the cooling load significantly. Compared to TRNSYS, the results from EnergyPlus show higher cooling loads during the night times, which can be caused by higher thermal capacitance. Nevertheless, this difference was improved significantly after optimizing the thermal model towards the measurement data using GenOpt.

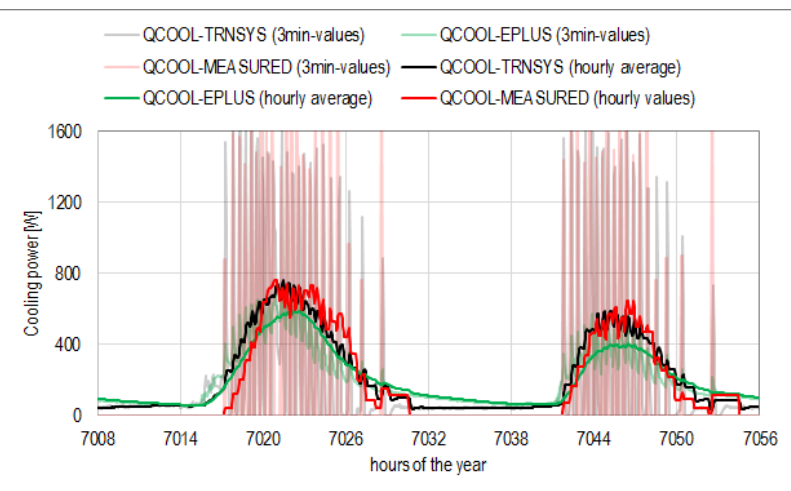

Figure 16: Instantaneous cooling load - shaded situation

For case 2 using shading blinds, Figure 16 shows the comparison for another 2-days trend. Also in this case, TRNSYS corresponds well with the measured values, while EnergyPlus is underestimating the cooling load. Still, however, both toolchain approaches show clearly the ability, to represent the measured situation with high accuracy.

\section{Conclusion}

With both proposed toolchain workflows, two powerful and highly flexible approaches for the detailed modelling of CFS have been shown and applied successfully on theoretical as well as real-case scenarios. Nevertheless, differences occurred in the theoretical comparison although all influencing model data and parameters have been adjusted. Reasons for this are due to different modelling routines and algorithms within the used tools. The gained results for the measurement comparison for thermal comparison as well as for daylighting results are promising, showing a high reliability of the models. Especially after the thermal model calibration, results improved significantly. Detailing the reasons for remaining differences will be part of further studies.

\section{Acknowledgement}

This study has been developed in the framework of the research activities of the project FACEcamp n. ITAT1039, funded by European Regional Development Fund and Interreg ITA AUT programme.

\section{References}

Bustamante, W., Vera, S., Ureta, F. (2015): Thermal and Lighting Performance of 5 Complex Fenestration Systems in a Semiarid Climate of Chile. In Energy Procedia 78, pp. 24942499. DOI: 10.1016/j.egypro.2015.11.249.

Frenzel, C.; Hiller, M. (2014): TRNSYSLIZARD Open source tool fpr Rhinoceros - Grasshopper. In Proceedings Bausim 2014, checked on 6/22/2015.

Gong, J., Kostro, A., Motamed, A., Schueler, A. (2016): Potential advantages of a multifunctional complex fenestration system with embedded micro-mirrors in daylighting. In Solar Energy 139, pp. 412-425. DOI: 10.1016/j.solener.2016.10.012.

Guglielmetti, R. (2015): Open Studio - Radiance Update. In 14th Radiance Workshop 2015.

Hauer, M.; Geisler-Moroder, D. (2017): Artlight 2.0 - a runtime optimized algorithm for coupled thermal and daylight simulation with TRNSYS and RADIANCE, pp. 701-710. DOI: $10.26868 / 25222708.2017 .181$.

Hiller, M.; Schöttl, P. (2014): Modellierung komplexer Verglasungssysteme in TRNSYS. In Proceedings Bausim 2014.

Karlsen, L.; Grozman, G.; Heiselberg, P.; Bryn, I. (2015): Integrated design of daylight, thermal comfort and energy demand with use of IDA ICE. In 7th Passivhus Norden Sustainable Cities and Buildings, checked on 9/7/2015.

Kirimtat, A.; Koyunbaba, B. K.; Chatzikonstantinou, I.; Sariyildiz, S. (2016): Review of simulation modeling for shading devices in buildings. In Renewable and Sustainable Energy Reviews 53, pp. 23-49. DOI: 10.1016/j.rser.2015.08.020

Lee, E., Geisler-Moroder, D., Ward, G. (2018): Modeling the direct sun component in buildings using matrix algebraic approaches: Methods and validation. In Solar Energy 160, pp. 380-395. DOI: 10.1016/j.solener.2017.12.029.

Loonen, R.C.G.M.; Favoino, F.; Hensen, J.L.M.; Overend, M. (2016): Review of current status, requirements and opportunities for building performance simulation of adaptive facades. In Journal of Building Performance Simulation 10 (2), pp. 205-223. DOI: 10.1080/19401493.2016.1152303.

Pyonchan, I.; Abderrezek, N.; Moncef, K. (2009): Estimation of lighting energy savings from daylighting. In Building and Environment 44 (3), pp. 509-514. DOI:

10.1016/j.buildenv.2008.04.016.

Santos, L., Leitão, A., Caldas, L. (2018): A comparison of two light-redirecting fenestration systems using a modified modeling technique for Radiance 3-phase method simulations. In Solar Energy 161, pp. 47-63. DOI: 10.1016/j.solener.2017.12.020.

Saxena, M.; Ward, G.; Perry, T.; Heschong, L.; Higa, R. (2010): Dynamic Radiance - Predicting annual Daylight with variable Fenestration optics using BSDFs. Available online at http://www.ibpsa.us/sites/default/files/publications/SB10DOC-TS08A-01-Saxena.pdf, checked on 9/17/2016.

International Organisation for Standardisation (2003) Thermal performance of windows, doors and shading devices Detailed calculations (EN ISO 15099) 